Pathologe 2008 · [Suppl 2] 29:129-134

DOI 10.1007/s00292-008-1047-6

(C) Springer Medizin Verlag 2008

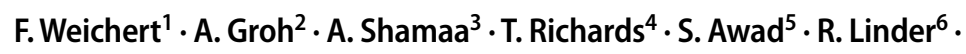
C.A. Landes ${ }^{7} \cdot$ M. Wagner ${ }^{8}$

${ }^{1}$ Fakultät für Informatik, Lehrstuhl für Graphische Systeme, Technische Universität Dortmund

2 Institut für Angewandte Mathematik, Saarbrücken

${ }^{3}$ Department of Oral Biology, Minia University, Minia, Ägypten

${ }^{4}$ University of the West Indies, Kingston, Jamaika

${ }^{5}$ Department of Maxillofacial Surgery, University of Mansoura, Ägypten

${ }^{6}$ Institut für Medizinische Informatik, Universität zu Lübeck

${ }^{7}$ Klinik für Mund-, Kiefer- und Plastische Gesichtschirurgie, Universitätsklinikum Frankfurt am Main

${ }^{8}$ Institut für Pathologie, Universität des Saarlandes, Saarbrücken

\title{
Signaltheoretische Analyse histologischer Daten im Ortsfrequenzraum
}

rung histologischer Schnittpräparate mit teilweise über $1000 \mathrm{zu}$ markierenden $\mathrm{Ob}$ jekten einen großen Arbeitsaufwand dar. Die manuelle Segmentierung eines einzelnen histologischen Schnittpräparates kann (je nach Zahl der relevanten Objekte) mehrere Stunden bis zu einigen Tagen dauern. Um die Bearbeitungszeit signifikant zu verkürzen, wird angestrebt, diesen Vorgang möglichst automatisch durch einen Segmentierungsalgorithmus durchführen zu können. Die meisten aktuellen Segmentierungssysteme erlauben eine Grobsegmentierung der Zelle, wobei teilweise eine Reduktion auf Graustufen erfolgt oder nur eine Detektion der Zellkerne [11]. Im Gegensatz zu den gradientenbasierten Verfahren versprechen Algorithmen unter Nutzung der Texturinformation eine nachhaltigere Segmentierungsoption. Dahingehend wird in dieser Arbeit eine erweiterte, signaltheoretisch motivierte Textursegmentierung mittels Wavelets genutzt. Ein ähnlicher Ansatz wird bei Zhang et al [11] beschrieben, allerdings wird hier eine abweichende Wavelet-Funktion eingesetzt, und es erfolgt nur eine Segmentierung von unspezifischen, künstlich generierten Texturen.

Die nachfolgende Betrachtung fokussiert sich auf die Segmentierung digitali- sierter arterieller Blutgefäße am Beispiel histologischer Schnittpräparate von Koronararterien, in denen $u$. a. die histologisch differenzierbaren Wandschichten Intima, Media und Adventitia durch signifikante Farbverläufe (Graustufen) oder eindeutige Texturen in den Präparaten zu erkennen sind. Das zugehörige A-priori-Wissen über anatomische Gegebenheiten und deren Repräsentation in der grafischen Darstellung kann in dem vorgestellten Ansatz für maschinelle Lernverfahren zur Modellbildung herangezogen und durch Merkmale auf Basis einer signaltheoretischen Extraktion umgesetzt werden. In der hier thematisierten Studie sollte untersucht werden, ob durch diese Herangehensweise histomorphologisch relevante Strukturen detektiert werden, beispielsweise in einer Hämatoxylin- und Eosin(HE-)Färbung von Gewebe (• Abb. 1a).

\section{Material und Methoden}

Die Ausgangsbasis der Bearbeitung sind digitalisierte Bilddatensätze mit einer durchschnittlichen Größe von $1500 \times 1500$ Pixeln, die HE-gefärbte histologische Schnittpräparate repräsentieren. Da präparationsbedingt von keiner eindeutigen Orientierung der relevanten
In Anlehnung an den Anwendungskontext stellt die manuelle Segmentie- 


\section{Hauptreferate: Systempathologie des Gewebes}

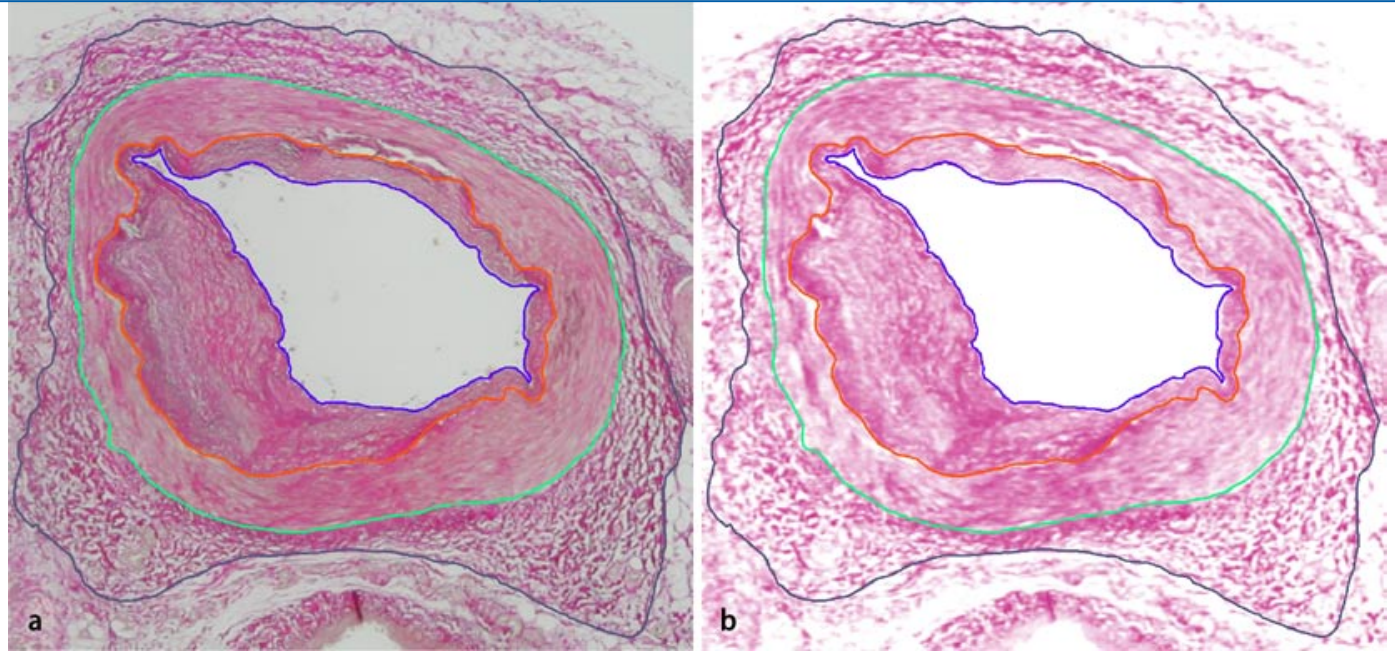

Abb. $1<$ Manuell segmentierte Binnenstrukturen einer Arterie; Darstellung $\mathbf{a}$ vor und $\mathbf{b}$ nach Anwendung einer Farbdekonvolution (HE-Färbung, Vergr. 25:1)

Objekte auf dem Objektträger und damit der Texturierung auszugehen ist, wird eine rotationssymmetrische Wavelet-Transformation eingesetzt, welche die Bestimmung von isotropen, also richtungsunabhängigen Merkmalen ermöglicht [10]. Allgemein, wie z. B. bei Keratinozyten, ist auch eine Betrachtung der Orientierung entsprechender Objekte im Gewebeverband relevant. Aufgrund der hohen Bildauflösung der digitalisierten histologischen Schnittpräparate war die Notwendigkeit einer effizienten Berechnung der Wavelet-Transformation notwendig. Umgesetzt wurde diese Anforderung durch die Berechnung der Wavelet-Transformation im Ortsfrequenzraum. Zur Integration des medizinischen Fachwissens in den Segmentierungs- und Klassifikationsablauf kommen überwachte maschinelle Lernverfahren, wie z. B. künstliche neuronale Netze (ANN; [3]) oder Support Vector Machines (SVM; [7]), zur Anwendung, die sich an manuell segmentierte histologische und zytologische Präparate adaptieren. Um den jeweiligen histologischen Anfärbemethoden gerecht zu werden, erfolgt in Abhängigkeit der Fragestellung im Vorverarbeitungsschritt eine Farbdekonvolution [2]. Die $\bullet$ Abb. 1b zeigt das Ergebnis dieser Operation auf das unter - Abb. 1a dargestellte histologische Schnittpräparat.

Den einleitenden Ausführungen folgend, kann die bildliche Darstellung eines Schnittpräparates als Funktion $f(x, y)$ mit $x, y \in \Re^{2}$ betrachtet werden, bei dem die Variablen $x$ respektive $y$ die horizontale und vertikale Ausrichtung repräsentieren. Im Hinblick auf eine geschlossene Darstel- lung bezeichnet in den weiteren Betrachtungen $f(\mathbf{x}) \in L^{2}\left(\Re^{2}\right)$, mit $\mathbf{x} \in \Re^{2}$ das 2-dimensionale (Bild-)Signal [1]. Unter Einbeziehung dieser Prämissen lässt sich die Definition der 2-dimensionalen Wavelet-Transformation wie folgt darstellen:

$$
\begin{aligned}
& \psi_{\mathbf{t}, s, \theta}(\mathbf{x})=\frac{1}{s} \psi\left(\boldsymbol{r}_{\theta}\left(\frac{\mathbf{x}-\mathbf{t}}{\mathrm{s}}\right)\right) \\
& \text { mit } s \in \Re \backslash 0, \mathbf{t} \in \Re^{2}
\end{aligned}
$$

$t$ bezeichnet in dieser Notation den Translationsvektor, $s$ den Skalierungsparameter und $\theta$ den Rotationswinkel. Unter Parametrisierung von $\theta$ wird der Rotationsoperator $r$ auf $x=(x, y)$ in der Form

$$
\begin{aligned}
& \mathbf{r}_{\theta}(\mathbf{x}) \\
& =(x \cos (\theta)-y \sin (\theta), x \sin (\theta) \\
& +y \cos (\theta)), \text { mit } 0 \leqslant \theta<2 \pi
\end{aligned}
$$

angewendet [1]. Gemäß der Rotationssensitivität des Mutterwavelets $\psi$ wird zwischen isotropen und anisotropen Wavelets unterschieden. Es ist hierbei anhand der einleitenden Problematik zu unterscheiden, ob im zu analysierenden (Bild-) Signal Merkmale detektiert werden sollen, die eine ausgezeichnete Richtungssensitivität aufweisen oder rotationsinvariant sind. Das bereits angesprochene Mexican Hat-Wavelet ist ein Repräsentant der 2-dimensionalen isotropen Wavelets, das wie folgt definiert ist:

$$
\psi(\mathbf{x})=\left(2-|\mathbf{x}|^{2}\right) e^{\left(-0.5|\mathbf{x}|^{2}\right)}
$$

Dieses auf Marr zurückgehende Wavelet liefert eine Punkt-bezogene Analyse, ohne Beeinflussung der Richtung [6]. Da die 2-dimensionale Wavelet-Transformation im Hinblick auf eine effektive Berech- nung über eine Multiplikation im OrtsFrequenz-Raum berechnet wird, ist das Wavelet entsprechend als Fourier-Transformierte anzugeben. Im Fall des Mexican Hat-Wavelets ergibt sich folgende Gleichung:

$$
\psi(\mathbf{x})=\frac{1}{\sqrt{2 \pi}} \mathbf{x} e^{\left(-0.5|\mathbf{x}|^{2}\right)}
$$

Die eigentliche (kontinuierliche) 2-dimensionale Wavelet-Transformation eines (Bild-)Signals $f$ ergibt sich somit als Korrelation der (Bild-)Funktion $f(x)$ mit dem normalisierten Wavelet $\psi \mathrm{zu}$

$W_{f}(\mathbf{x}, s, \theta)$

$=s \int_{\Re^{2}} f(\mathbf{x}) \psi^{*}\left(\mathbf{r}_{-\theta}\left(\frac{\mathbf{x}-\mathbf{t}}{\mathrm{s}}\right)\right) d^{2} \mathbf{x}$.

Wie bereits einleitend ausgeführt, repräsentiert $t$ den Translationsvektor, $s$ den Skalierungsparameter und $\theta$ den Rotationswinkel. Unter Ausnutzung des Faltungstheorems kann die Gleichung (5) in eine Multiplikation der Fourier-Transformierten von $f$ und $\Psi, F$ und $\Psi$ überführt werden $[1,10]$.

$$
\begin{aligned}
& W_{f}(\mathbf{t}, s, \theta)=\frac{1}{s} \int_{\Re^{2}} F(\mathbf{k}) \psi^{*} \\
& \left(s \mathbf{r}_{-\theta}(\mathbf{k})\right) e^{2 \pi \cdot i \cdot \mathbf{t} \cdot \mathbf{k}} d^{2} \mathbf{k} .
\end{aligned}
$$

Bei der weitergehenden Anwendung der 2-dimensionalen Wavelet-Transformation $W_{\mathrm{f}}(t, s, \theta)$ ist $\mathrm{zu}$ beachten, dass die Transformation in dieser Form von $4 \mathrm{~Pa}$ rametern abhängig wäre - 2 Parameter bestimmen die Translation $\left(\mathbf{t} \in \Re^{2}\right), 1 \mathrm{~Pa}$ rameter die Skalierung $(s)$ und einer die Rotation $(\theta)$. Wird, wie im vorliegenden Fall, von einer rotationsinvarianten Sichtweise ausgegangen, kann der Rotationsparameter $\theta$ in Gleichung (6) vernachlässigt 
werden, was mit einer Reduktion auf $3 \mathrm{~Pa}-$ rameter verbunden ist [10]. Diese Sichtweise wird in der weiteren Darstellung verfolgt. Sie liefert neben der Rotationsinvarianz den Vorteil einer anschaulichen Darstellung, die in Assoziation mit der Verwendung der Wavelet-Koeffizienten steht. Im Sinne der effizienten Berechenbarkeit wird zur Diskretisierung die dyadische Abtastung genutzt. Die hierzu notwendige Skalierungsvariable $s$ wird in Abhängigkeit zur Auswertung der $k$-ten Skalierungsebene gemäß

$s_{k}$

$=s_{0} \cdot s_{B}^{k \Delta s}$ mit $s_{k} \leqslant s_{\max }, s_{B}>1$

definiert, wobei $s_{\mathrm{O}}$ um eine parametrisierbare Potenz der Basis $s_{\mathrm{B}}$ gesteckt wird. $\Delta s$ legt weiterhin die Skalierungsdiskretisierung fest. Über $s_{\mathrm{O}}$ und $s_{\max }$ kann der Ausschnitt aus der Gesamtheit der Skalierungsebenen $k$ bestimmt werden. Die Anzahl der möglichen Skalierungsstufen

$|k|=\frac{\log _{s_{B}}(N / 2)}{\Delta s}$

wird durch Parametrisierung von $s_{\mathrm{B}}$ und $\Delta s$ sowie der maximalen Auflösung des Bildes $N$ initial bestimmt. Exemplarisch bedeutet dieses, dass bei einem histologischen Schnittpräparat mit einer Auflösung von $1500 \times 1500$ Pixeln, einer Basis $s_{\mathrm{B}}=1.3$ und einer Skalierungsdiskretisierung von $\Delta s=0.4$ maximal 63 Skalierungsebenen möglich sind. Eine Abwandlung auf nichtquadratische Bilder ist implizit auch möglich, sei aber im Sinne einer vereinfachten Darstellung nicht weiter ausgeführt.

In Anlehnung an die obigen Ausführungen kann die Wavelet-Transformation auch als Bandpass-Filter verstanden werden, bei dem das Wavelet ein definiertes Frequenzband aus dem Spektrum extrahiert. In tieferen Frequenzen resultiert dieses in extrem schmalen Frequenzbändern (hohe Frequenzauflösung), bei höheren Frequenzen wird das herausgefilterte Band breiter, die Frequenzauflösung nimmt somit ab. Die Wavelet-Transformation liefert je nach Quantisierung des Skalierungsfaktors eine bestimmte Anzahl von Transformationsbildern (Bandaufspaltungen). Demzufolge enthält jedes Transformationsbild nur den Bildausschnitt aus dem ursprünglichen Fre- quenzspektrum, der von dem skalierten Wavelet erfasst wird. Zur Verdeutlichung der bisherigen Zusammenhänge stellt die - Abb. 2 den Zusammenhang zwischen einem histologischen Schnittpräparat und der Repräsentation als Wavelet-Transformierte dar. Die Transformationsbilder zeigen das aus $\bullet$ Abb. 1 bekannte histologische Schnittpräparat in unterschiedlichen Skalierungsstufen der Wavelet-Transformation. Erkennbar ist, dass die Konturen durch den geringeren Hochpassanteil verschwommen sind, eine Lokalisation jedoch weiterhin gegeben ist. Speziell in tieferen Frequenzbereichen ist eine augenfällige Korrelation mit den anatomischen Strukturen erkennbar.

Bei der Betrachtung der bisher bestimmten Merkmale für eine automatische Segmentierung lässt sich festhalten, dass jeder Punkt $(x, y)$ im histologischen Schnittpräparat durch einen Vektor im Frequenzraum repräsentiert wird, dessen Dimension der Anzahl der Skalierungsstufen $k$ entspricht. Ausgehend von der angedachten (semi)automatischen Segmentierung ist es das Anstreben eines maschinellen Lernverfahrens, gemeinsame Merkmale für Bildpunkte zu finden, sofern sie einer segmentierten Gruppe entsprechen. Dieses Vorgehen ist dadurch motiviert, dass alle Objekte einer durch Klassifizieren entstandenen Gruppe (medizinisches Kompartiment) mindestens 1 gemeinsames Merkmal besitzen, das die Objekte anderer Klassen nicht besitzen, ansonsten wären sie bei der manuellen Segmentierung nicht zusammengefasst worden. Maschinelles Lernen hat damit das Ziel eine Klassifikation zu ermöglichen, in dem gemeinsame Merkmale einer Gruppe bzw. abgrenzende Merkmale zwischen verschiedenen Gruppen detektiert werden. Im Sinne der Anwendung entsprechen die Gruppen den medizinischen Kompartimenten (Lumen, Intima, Media), die auch durch Segmentierung differenziert wurden, Merkmale sind in der Konsequenz Kennzahlen, die aus der Wavelet-Zerlegung resultieren. Die Idee der Klassifikation wird durch maschinelle Lernverfahren umgesetzt diese detektieren initial pro Struktur eine gemeinsame Merkmalskomposition und wenden sie auf unbekannte Daten an. Da für die weitere Diskussion ein detaillier-
Pathologe 2008 · [Suppl 2] 29:129-134

DOI 10.1007/s00292-008-1047-6

(c) Springer Medizin Verlag 2008

F. Weichert · A. Groh · A. Shamaa ·

T. Richards · S. Awd · R. Linder · C.A. Landes . M. Wagner

Signaltheoretische Analyse histologischer Daten im Ortsfrequenzraum

\section{Zusammenfassung}

Diese Arbeit beschreibt einen neuen Algorithmus zur (semi)automatischen Segmentierung histologischer und zytologischer Präparate. Bedingt durch den Umstand, dass derartige Präparate teilweise nur wenige kontrastreiche Areale und kaum scharfe Konturen aufweisen, sind klassische Segmentierungsverfahren wenig Erfolg versprechend, die relevanten Strukturen zu detektieren. Daher wird angestrebt, die gesuchten Strukturen anhand ihrer Texturierung auf Basis der Wavelet-Transformation zu identifizieren. Die ermittelten Merkmale dienen als Basis für ei-

ne nachgeschaltete Klassifikation mittels maschineller Lernverfahren.

Schlüsselwörter

Systembiologie · Segmentierung · Klassifikation - Wavelets · Maschinelles Lernen

Analysis of histological datasets by signal processing methods

Abstract

In the present study, a semi-automatic segmentation and classification algorithm is proposed for the analysis of histological and cytological images. In view of the fact that histological and cytological images usually exhibit poor contrast and blurred outlines, classical segmentation algorithms often fail to detect relevant structures. A new algorithm for texture segmentation based on signal processing methods in combination with machine learning techniques was therefore developed.

Keywords

Systems biology - Segmentation - Classification Wavelets - Machine learning 


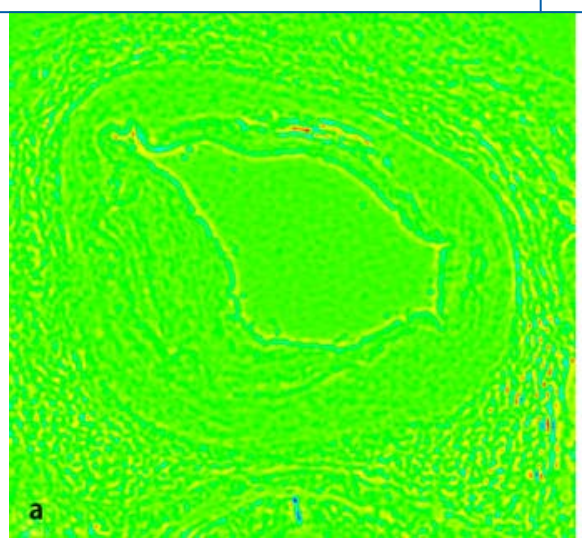

\section{Hauptreferate: Systempathologie des Gewebes}

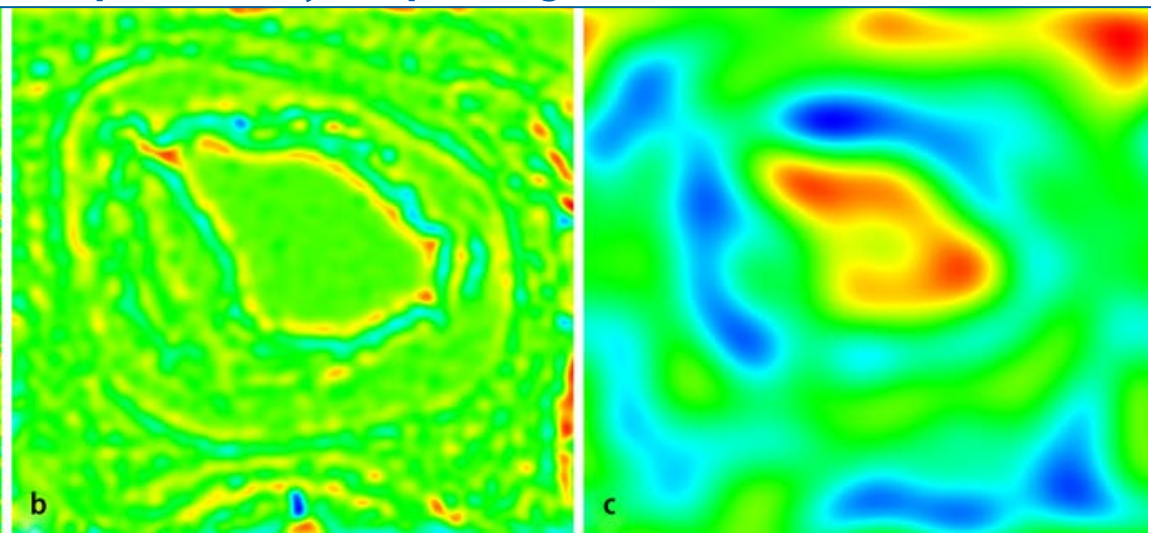

Abb. $2 \Delta$ Transformationsbilder des in Abb. 1 dargestellten histologischen Schnittpräparats in unterschiedlichen Skalierungsstufen der Wavelet-Transformation: $\mathbf{a}$ hohe, $\mathbf{b}$ mittlere und $\mathbf{c}$ tiefe Frequenzen

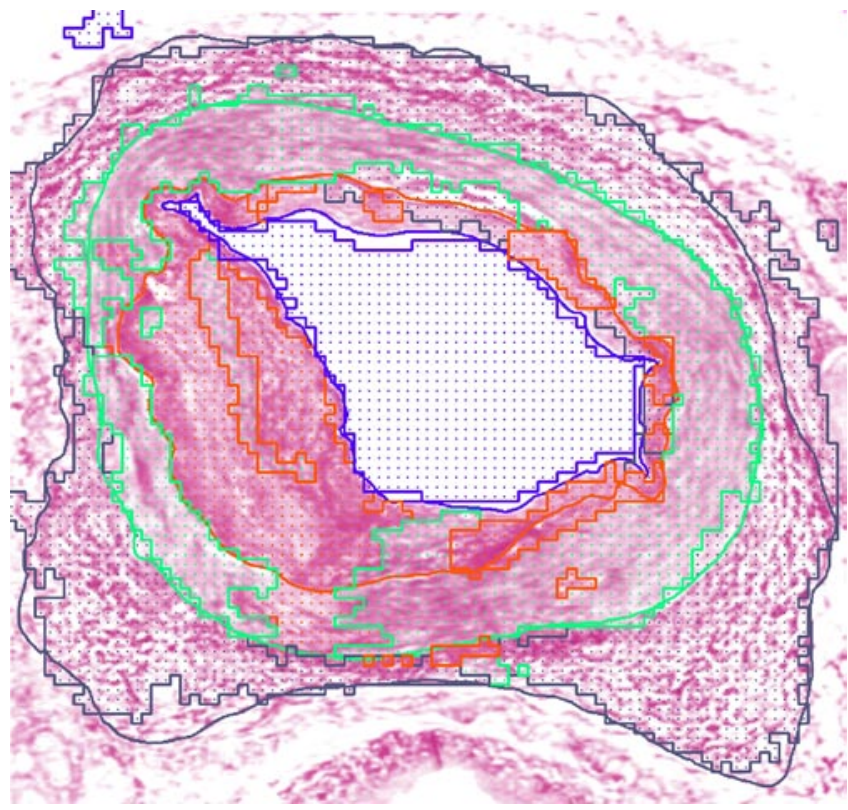

Abb. $3 \Delta$ Vergleichende Darstellung der Ergebnisse einer manuellen und (semi)automatischen Segmentierung. Letztere sind mit einer gepunkteten Texturierung dargestellt (Ausgangsmaterial: Abb. 1a)

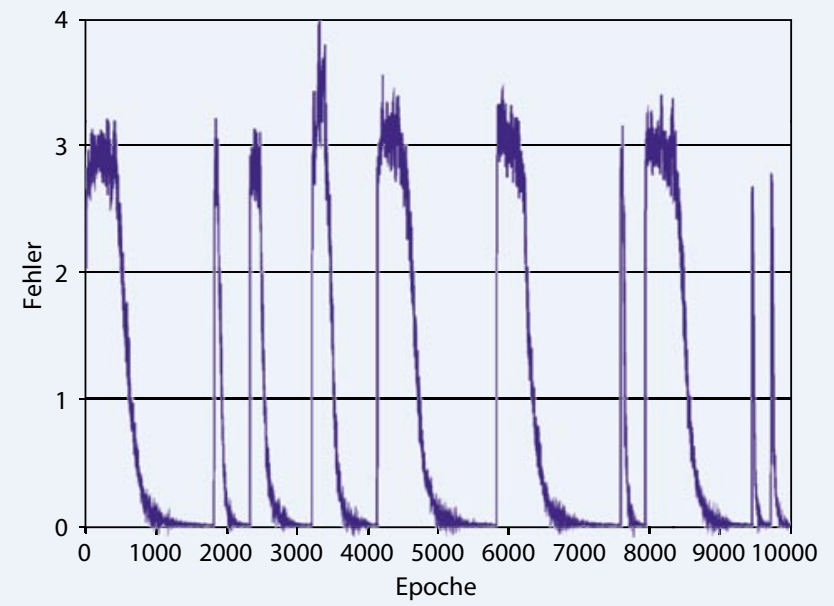

Abb. $4 \Delta$ Darstellung der Lernkurve einer Support-Vector-Maschine. Die Merkmale resultierten aus den Merkmalen der Wavelet-Transformation. Erkennbar ist das unterschiedliche Adaptionsverhalten der SVM bezüglich der unterschiedlichen Kombinationen zwischen den anatomischen Strukturen teres Verständnis zur Theorie maschineller Lernverfahren nur eingeschränkt notwendig ist und auch über die Darstellung dieser Ausführungen hinausgeht, sei hierzu auf die Literatur verwiesen $[1,7]$.

Zur Verifikation des obigen Ansatzes wurden die zur Verfügung stehenden histologischen Schnittpräparate in eine Lernund Testgruppe separiert und gemäß unterschiedlichen Ansätzen evaluiert. Neben der Variation der Skalen wurde das Verhältnis zwischen der Lern- und Testgruppe variiert, eine Beschränkung auf ausgezeichnete anatomische Strukturen vorgenommen oder wahlweise als maschinelles Lernverfahren ein künstliches neuronales Netz bzw. eine Support-Vector-Machine genutzt. Ergänzend kam bei einigen Versuchen die Farbdekonvolution zur Anwendung. Die nachfolgende Auswertung ist dahingehend exemplarisch zu sehen: In dieser werden alle anatomischen Strukturen beachtet, eine Farbdekonvolution wird durchgeführt, und als maschinelles Lernverfahren kommt eine Support-VectorMachine zum Tragen. Im Hinblick auf eine simplifizierende Darstellung wurde jeweils nur ein histologisches Schnittpräparat als Lern- respektive Testdatensatz genutzt.

\section{Ergebnisse}

Basierend auf den obigen Ausführungen zur Vorgehensweise der Verifikation stellt - Abb. 3 die Ergebnisse dar. Erkennbar ist die recht gute Übereinstimmung zwischen der manuellen und automatischen Segmentierung. Sowohl das Lumen als auch die Intima-Media-Region werden augenfällig korrekt detektiert. Zwar sind fehlerhafte Einschlüsse in der Intima-Media-Region oder der Adventitia erkennbar, doch liegen sie in Abhängigkeit von der Fragestellung im tolerierbaren Bereich. Eine vergleichbare Aussage spiegelt sich auch in der Anwendung der SVM wieder. Zu diesem Kontext zeigt $\bullet$ Abb. 4 das Verhalten 

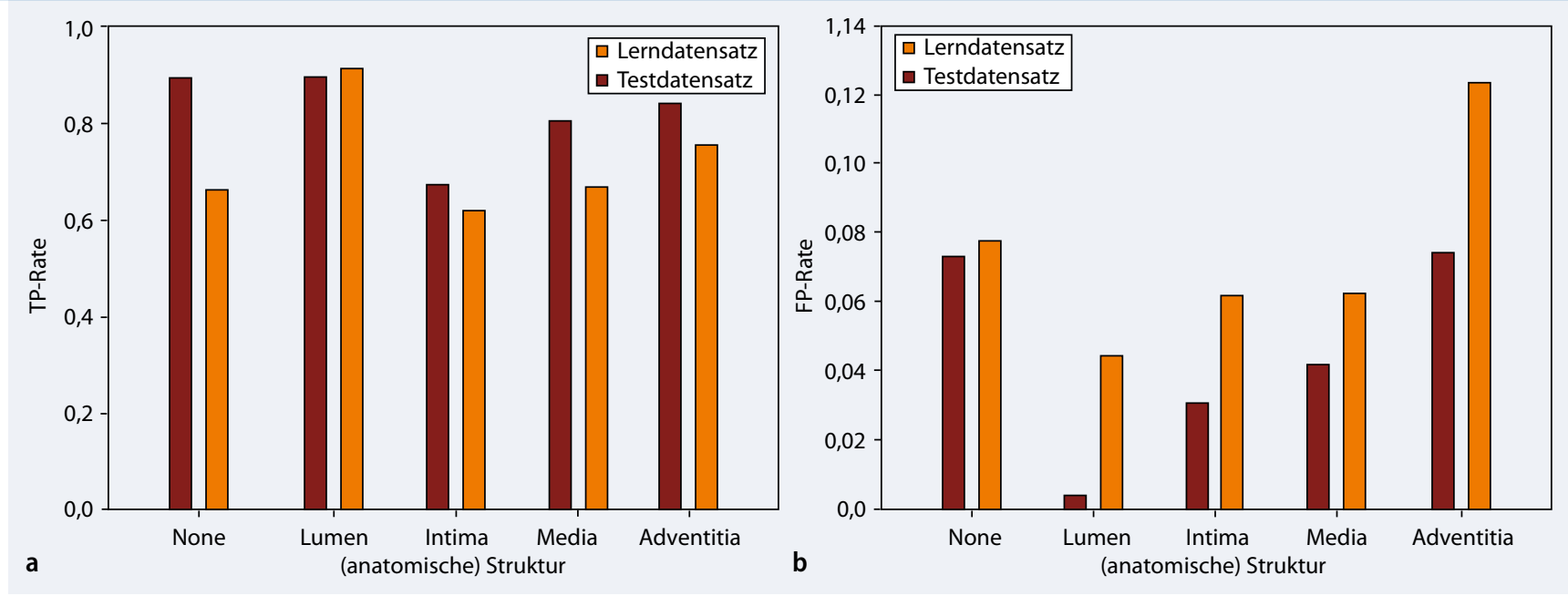

Abb. $5 \Delta$ Vergleichende statistische Auswertung zwischen einem Lern- und Testdatensatz. Als Merkmal der Güte ist die jeweilige "True Positive" (TP-) und "False Poistive" (FP-) Rate einer anatomischen Struktur grafisch aufgetragen

der Lernkurven. Da es sich bei der SVM um ein duales Lernverfahren handelt, werden immer paarweise Strukturen gelernt und die Ergebnisse nachfolgend in Beziehung gesetzt. Aus dieser Vorgehensweise resultieren auch die inhärenten 10 Lernkurven - sie entsprechen den unterschiedlichen Kombinationen der anatomischen Strukturen. Beispielsweise zeigt die erste Teilkurve die Möglichkeit der Trennung vom Lumen zum Hintergrund. In Anbetracht der Tatsache, dass in beiden Strukturen ausgeprägtere homogene Bereiche (im Sinne der Wavelet-Transformation tiefere Frequenzen) ersichtlich sind, gestaltet sich diese Differenzierung schwieriger. Insgesamt zeigt aber gerade die vergleichende statistische Auswertung innerhalb der $\bullet$ Abb. 5 das Potenzial des Ansatzes zur (semi)automatischen Segmentierung - eine nachhaltige Unterscheidung zwischen dem Lern- und Testdatensatz ist nicht manifestierbar.

\section{Diskussion}

Initial wurde die Herangehensweise des beschriebenen Verfahrens zur Segmentierung und Klassifikation intravaskulärer Ultraschalldaten konzipiert. Bedingt durch gewebecharakteristische Reflexionen und Absorptionen des Ultraschallsignals ist es möglich, die arterielle Morphologie in derartigen Datensätzen zu detektieren, um eine Bestrahlungsplanung bei intravaskulärer Brachytherapie vorzubereiten [9]. Ausgangsbasis des Verfahrens ist die Beobachtung, dass verschie- dene anatomische Strukturen auch durch klar zu differenzierende texturelle Ausprägungen repräsentiert werden. Wie im vorhergehenden Abschnitt dargelegt, zeigt der vorgestellte Ansatz ein ausgeglichenes Verhältnis zwischen Spezialisierung und Generalisierung, sodass er geeignet erscheint, auch Daten aus anderen Quellen suffizient verarbeiten zu können. Signaltheoretische Verfahren wie die angesprochene Wavelet-Transformation sind in der Lage, eine kompakte Darstellung der definierten texturellen Ausprägungen anatomischer Strukturen zu liefern, und erlauben maschinellen Lernverfahren, diese Merkmale geeignet zu nutzen. Durch die Integration der überwachten Lernverfahren ist es möglich, das A-priori-Wissen der medizinischen Experten („Expertenwissen “) in das Verfahren zu integrieren. Speziell dieser Absatz erlaubt eine weitreichende Nutzbarkeit des Verfahrens. So können, ausgehend von einer vorliegenden automatischen Segmentierung arterieller Blutgefäße und evtl. vorhandener Stents, umfassende Blutströmungssimulationen initiiert werden, um die Strömungsdynamik besser analysieren zu können. Das Verfahren kann beispielsweise auch zur (semi)automatischen Bearbeitung digitalisierter histologischer Großflächenschnittpräparate aus der Lippen-, Kiefer- und Gaumenregion genutzt werden, um als Basis für eine 3-dimensionale Rekonstruktion zu fungieren [4]. Neben der aufgezeigten Verwendung liegt ein weiterer Aspekt auch in der Nutzbarkeit innerhalb einer systembiologischen
Betrachtungsdomäne. Systembiologie hat zum Ziel, ausgehend von statischen und statistischen Kenngrößen, Modelle abzuleiten, um die Veränderlichkeit histologischer Strukturen rechnerseitig nachzubilden [8]. Ausgangsbasis dieser Überlegungen sind die Informationen der Segmentierung [9].

\section{Fazit für die Praxis}

\section{Die Kombination aus signaltheore-} tischer Analyse, im vorliegenden Fall über die Wavelet-Transformation und maschinellen Lernverfahren, liefert einen neuen, vielversprechenden Ansatz zur (semi)automatischen Segmentierung histologischer und zytologischer Präparate, aber auch thematisch angrenzender Betrachtungen. Über die variable Parametrisierung ist eine nachhaltige Nutzung in unterschiedlichen Anwendungsdomänen der Histologie und Zytologie denkbar. Entscheidend für die Akzeptanz des Verfahrens durch Mediziner wird auch die intuitive Bestimmung geeigneter Parameter sein. Trotz dieser marginalen Einschränkung kann der vorgestellte Algorithmus als Bindeglied in der Systembiologie fungieren, da das Verfahren histologische Schnittpräparate durch die Bereitstellung einer Segmentierung entscheidend ergänzt und damit mathematischen Modellen der Systembiologie einen Zugang zu realen Daten liefert. 


\section{Hier steht eine Anzeige.}

\section{Springer}

\section{Korrespondenzadresse}

\section{F. Weichert}

Fakultät für Informatik, Lehrstuhl für Graphische Systeme, Technische Universität Dortmund, Otto-Hahn-Straße 16, 44221 Dortmund

frank.weichert@tu-dortmund.de

Interessenkonflikt. Der korrespondierende Autor gibt an, dass kein Interessenkonflikt besteht.

\section{Literatur}

1. Antoine JP (1996) Two-dimensional directional wavelets and image processing. In Proc IWISP'96: 53-60

2. Berger C, Koeijer J, Glas W, Madhuizen H (2006) Color separation in forensic iomage processing. J Forensic Sci 51: 100-102

3. Haykin S (1998) Neural Networks: a comprehensive foundation, 2nd edn. Prentice Hall

4. Landes C, Weichert F, Geis P et al. (2006) Evaluation of two 3D virtual computer reconstructions for comparison of cleft lip and palate to normal fetal microanatomy. Anat Rec A Discov Mol Cell Evol Biol 288: 248-262

5. Langheinrich AC, Bohle RM, Greschus $S$ et al. (2004) Atherosclerotic lesions at micro CT: feasibility for analysis of coronary artery wall in autopsy specimens. Radiology 231: 675-681

6. Marr D (1982) Vision. W.H. Freeman, San Francisco

7. Vapnik V (1995) The nature of statistical learning theory. Springer, Berlin Heidelberg New York

8. Wagner M, Zamelczyk-Pajewska M, Landes C et al. (2006) Simulating soft data to make soft data applicable to simulation. In Vivo 20:49-54

9. Weichert F, Wawro M, Müller H, Wilke C (2004) A 3D cardiovascular model for brachytherapy planning based on biplane angiography and intravascular ultrasound. Methods Inf Med 43: 398-402

10. Wouwer de G (1998) Wavelets for multiscale texture analysis. $\mathrm{PhD}$ thesis, Universität Antwerpen

11. Zhang J, Tan T, Ma L (2002) Invariant texture segmentation via circular gabor filters. Proceedings of the 16th international conference on pattern recognition, 2: 901-904 